Pramāna, Vol. 14, No. 5, May 1980, pp. 363-371, @ Printed in India.

\title{
A solid state pulsed NMR spectrometer*
}

\author{
R K SHENOY ${ }^{\dagger}$, J RAMAKRISHNA and $K$ R JEFFREY ${ }^{\dagger \dagger}$ \\ Department of Physics, Indian Institute of Science Bangalore, 560012 , India \\ †Present Address: Department of Physics, University of Waterloo, Waterloo, Canada \\ $\dagger+$ Department of Physics, University of Guelph, Guelph, Ontario, Canada \\ MS received 5 February 1980
}

\begin{abstract}
A $10 \mathrm{MHz}$ pulsed NMR spectrometer, built using mostly solid state devices, is described. The pulse programmer provides 2-pulse, 3-pulse, saturation burst and Carr-Purcell sequences both in repetitive and manual modes of operation. The transmitter has a maximum power output of $\sim 2 \mathrm{~kW}$ with a $75 \Omega$ output impedance termination. The total gain of the receiver system is around $120 \mathrm{~dB}$ with a minimum band width of $2 \mathrm{MHz}$. The recovery time of the receiver is $\sim 7 \mu \mathrm{sec}$. A two-channel boxcar integrator capable of working in the single channel, differential and double boxcar modes provides signal to noise ratio improvement. The sensitivity and the linearity of the boxcar integrator are $\sim 2 \mathrm{mV}$ and $\sim 0.1 \%$ respectively.
\end{abstract}

Keywords. Pulsed NMR spectrometer; pulse programmer; gated transmitter; video amplifier; quench circuit; box car integrator.

\section{Introduction}

Though transient signals in nuclear magnetic resonance (NMR) were observed (Hahn 1950a) a few years after the first detection of NMR in bulk matter, relaxation studies employing pulse techniques became popular only in the last decade. Commercial pulsed NMR spectrometers are available today, but they are very expensive. In this paper we describe a home-made pulsed NMR spectrometer employing solid state devices, mostly integrated circuits wherever convenient. The spectrometer meets most, if not all, of the requirements specified by Farrar and Becker (1971). These requirements may be relaxed selectively depending upon the particular experiment under study.

Preliminary reports of this work at various stages of development have already appeared (Shenoy and Ramakrishna 1975, 1977 and 1979).

\section{The pulsed NMR spectrometer}

e block diagram of the pulse spectrometer is given in figure 1. The $10 \mathrm{MHz}$ output from the RF unit is modulated by the pulses from the pulse programmer. The pulse modulated RF is then power-amplified and coupled to the sample through a single coil series-tuned probe. The transient signal from the sample at the end of the transmitter pulse, is amplified by a preamplifier and a tuned amplifier. The signal is then

*Research supported by a grant from the Department of Atomic Energy, Government of India. 


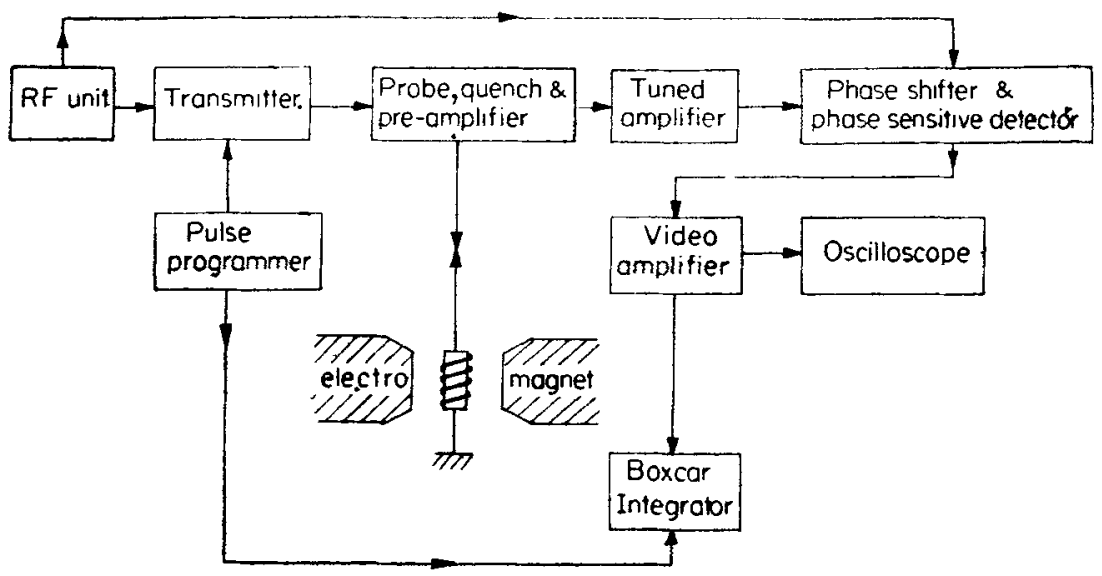

Figure 1. Block diagram of the pulsed NMR spectrometer.

phase detected and amplified further by a video amplifier. At this point the signal may either be displayed on an oscilloscope or may be measured using a boxcar integrator. The individual units of the spectrometer are discussed below.

\subsection{The digital pulse programmer}

The pulse programmer has been designed and constructed using digital IC's and the circuit details have already been published (Shenoy et al.1976). It has a crystalcontrolled time-base and is programmed to generate 2-pulse, 3-pulse, saturation burst (SB) and Carr-Purcell (CP) sequences. The pulse widths and the delays can be varied independently. The resolution achieved in these parameters is $0.2 \mu$ sec. The number of pulses in the SB and the CP sequences can be varied from 1-99. Relaxation times may be measured over a range of $100 \mu \mathrm{sec}$ to $25 \mathrm{sec}$. Times larger than $25 \mathrm{sec}$ can be measured by extending the time base.

\subsection{The RF unit}

The RF unit (figure 2) has a crystal-controlled oscillator working at $10.00027 \mathrm{MHz}$ followed by an emitter follower. The output ( $3 \mathrm{v}$ into $50 \mathrm{ohms}$ ) is connected to power divider (North Hills 50-201 RA), which gives two in-phase but isolated

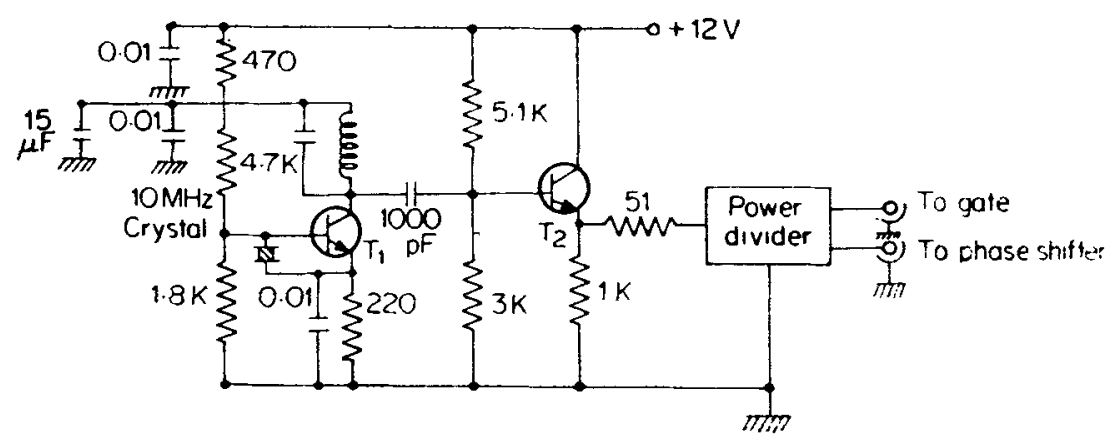

Figure 2. RF unit (crystal oscillator and power divider) $T_{1}, T_{2}$ are 2N2369, 
( $\sim 50 \mathrm{~dB}$ ) outputs. One is used as the RF input to the transmitter and the other as the reference for the phase sensitive detector.

\subsection{Gated iransmitter}

The RF from the source is gated and power amplified by the transmitter before coupling to the sample coil. Since there should be no RF power reaching the sample coil during the off-time of the gate, the carrier suppression ratio should be as high as possible. The gated transmitter has three stages: (i) an RF gate (ii) a gated driver (medium power amplifier) and (iii) a gated power amplifier.

Figure 3 shows the RF gate and the gated driver amplifier. Three separate circuits were tried as possible gates: (a) MC 1445 (Motorola); a gated video amplifier having a $60 \mathrm{~dB}$ RF suppression during the off time, (b) 76514 (Texas Instruments); a doublebalanced mixer with a $90 \mathrm{~dB}$ suppression and (c) 74H00 (Signetics); a quadruple 2 input NAND gate. The most successful was the 74H00 which gave a sharp RF pulse of $4 \mathrm{~V}$ amplitude with good rise and fall times (10 ns typically) and a high suppression ratio $(\sim 100 \mathrm{~dB})$ during the off-time of the pulse. All the four gates in the $74 \mathrm{H} 00$ have been used for maximizing the RF suppression.

An RCA 2N3375 RF power transistor has been used as the driver amplifier (figure 3). The transistor is held at cut-off with $-5 \mathrm{~V}$ on the base and d.c. pulses from the programmer, applied at the base, drive the transistor into the active region only during the ON time of the RF pulse. Thus, the pulsed RF from the RF gate is amplified only during the ON time of the synchronous gating pulse and RF leakage during the OFF time is thus further suppressed. $L_{1}$ and $L_{2}$ are adjusted to match the input and the output impedances of the preceding and the following stages respectively. The driver amplifier gives an output of $\sim 20 \mathrm{~V} \mathrm{pp}$ into 50 ohms load.

The output power amplifier consists of an RCA 3E29 dual tetrode used in the push-pull configuration (figure 4). The grids are biased at $-150 \mathrm{~V}$, holding the tube well below cut-off. The grids are gated through the wideband transformer (North Hills NH0900B) by the synchronous pulses which were amplified by a pulse amplifier (figure 4) similar to the one used by Lowe and Tarr (1968). Normally the transistor $T_{2}$ is not conducting. A positive going pulse at the input turns $T_{1}$ on. This switches

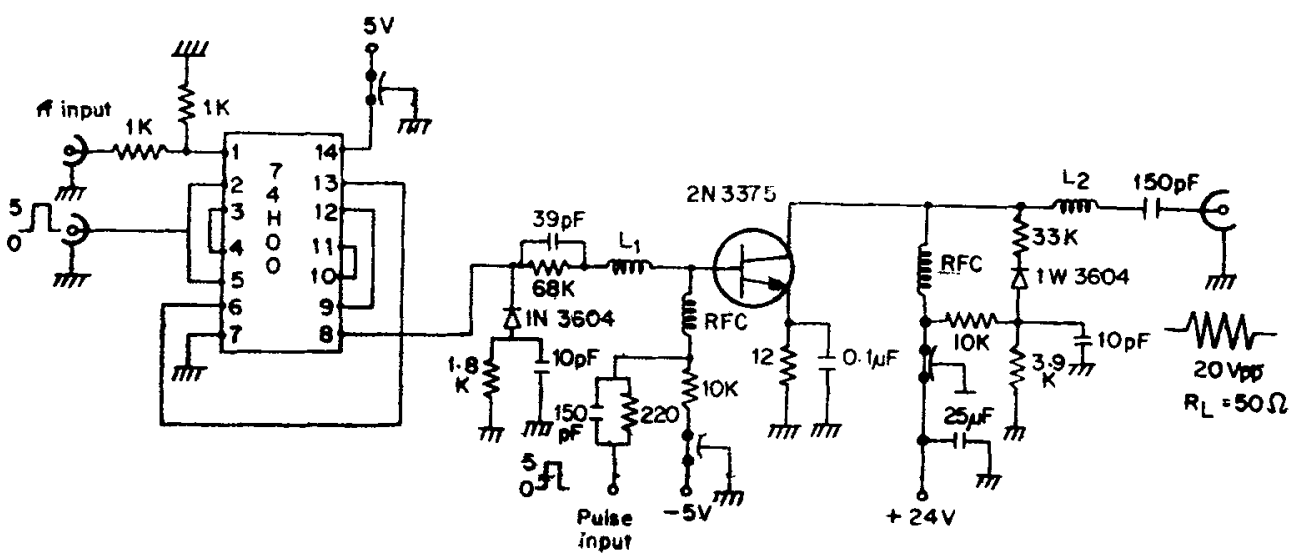

Figure 3. Gate and medium power amplifier. 


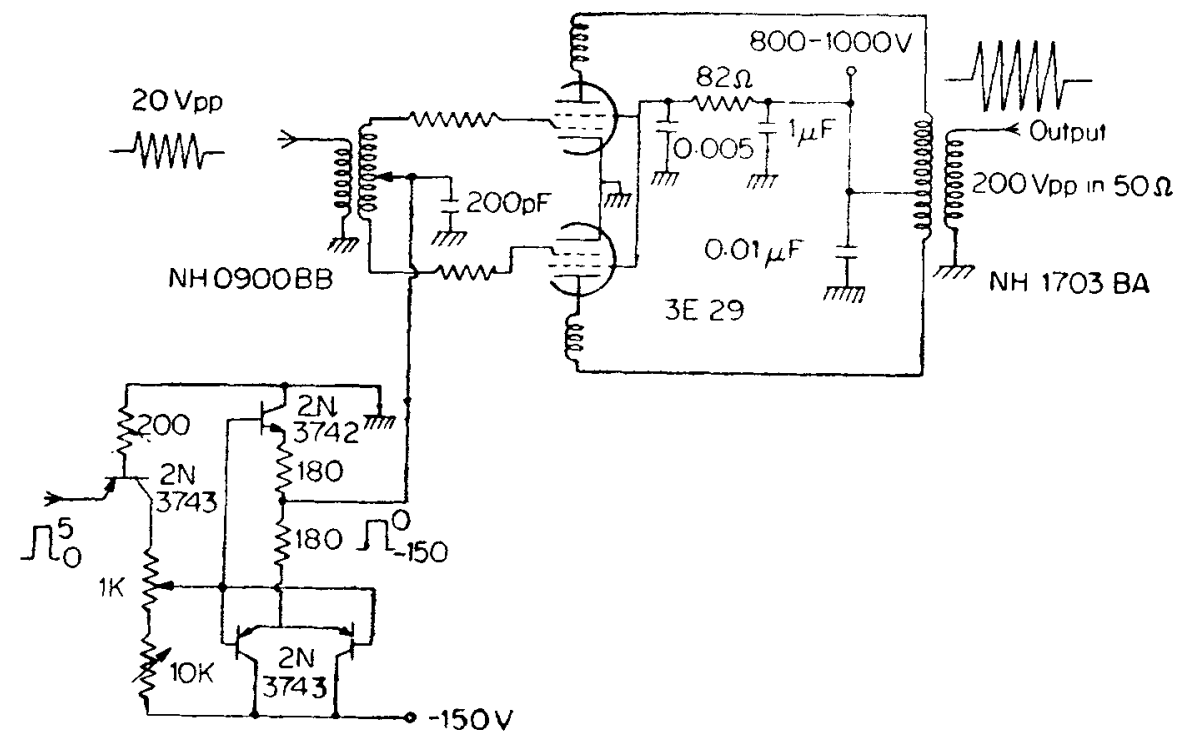

Figure 4. Power amplifier (final stage).

the voltage at the bases of $T_{2}, T_{3}$ and $T_{4}$ from -150 to $0 \mathrm{~V}$. $T_{2}$ and $T_{3}$ which were conducting hard earlier, turn OFF, whereas $T_{4}$ which was OFF earlier starts conducting. The output switches from $-150 \mathrm{~V}$ to nearly $0 \mathrm{~V}$. The $1 \mathrm{~K}$ and $10 \mathrm{~K}$ potentiometers at the bases of $T_{2}, T_{3}$ and $T_{4}$ are used to set the voltage at the grids of the 3E29 during the RF pulse.

The output of the push-pull amplifier is taken through another wide band transformer (North Hills NH1703BA) having an output impedance of 75 ohms. The transmitter RF pulse amplitude is variable from $400-600 \mathrm{~V}$.

Every stage of the transmitter is separately shielded to minimise RF leakage. The ON/OFF ratio is $\sim 10^{7}$. The final rise and fall times of the RF pulse are about $0.5 \mu \mathrm{sec}$.

\subsection{Receiver}

In NMR, the response to a pulsed excitation is a transient, the time duration of which is determined by the spin-spin relaxation time of the sample. In liquids, the spin-spin relaxation times are generally of the same order of magnitude as the spinlattice relaxation times and range from a few $\mathrm{m}$ sec to sec. However, in solids spinspin relaxation times are only a few $\mu$ sec and are very much shorter than spin lattice relaxation times. Hence, the transient signals, generally known as free-induction signals, are shortlived and it is, therefore, necessary to have a broadband receiver with a short recovery time well-protected from transmitter pulses. Usually the signal level at the sample coil is of the order of a few microvolts, and the signal has to be amplified sufficiently before it could be displayed. At resonance, the signal frequency is the same as that of the RF source and so all the amplifiers before detection should have the resonance frequency as their centre frequency. Altogether there are three units in the receiver system: (i) the preamplifier, (ii) the tuned amplifier and (iii) the phase-sensitive detector. 


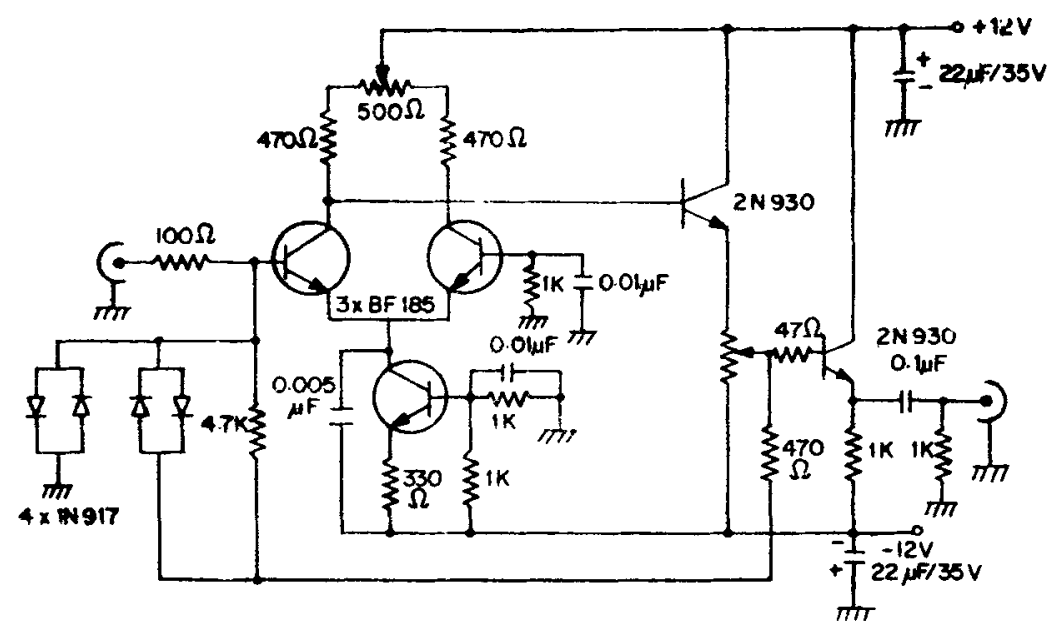

Figure 5. Fast recovery pre-amplifier.

2.4a. The preamplifier. The fast recovery preamplifier of Ramadan et al (1974) is modified to suit locally available transistors (figure 5). The limiter in the fcedback loop protects the receiver from excessive loading due to high power RF pulses. The baseline level adjustment ( $1 \mathrm{~K}$ variable resistor), allows the baseline to be placed symmetrically with respect to the positive and negative saturation limits. The problem of the baseline shift, after detection, due to asymmetry of saturation together with the AC coupling is actually faced after the detection of the signal. To overcome this problem, the same circuit is also used in the video amplifier after detection. The preamplifier has a gain of approximately $20 \mathrm{~dB}$, a bandwidth of $1 \mathrm{kHz}$ to 15 $\mathrm{MHz}$ and a recovery time of $2 \mu \mathrm{sec}$.

2.4b. The tuned amplifier. Further RF amplification is achieved by using a tuned amplifier. This consists of three tuned MOSFET (RCA 40673) stages, each having $\sim 20 \mathrm{~dB}$ gain, followed by a source follower (figure 6). A bandwidth of $2 \mathrm{MHz}$ is obtained by stagger tuning the amplifier stages. Though the preamplifier has a recovery time of $2 \mu \mathrm{sec}$, the RF leakage can still saturate the tuned amplifier. The blocking effect of capacitive interstage coupling (Adduci et al 1976) following the RF pulse increases the recovery time of the tuned amplifier. This has been eli-

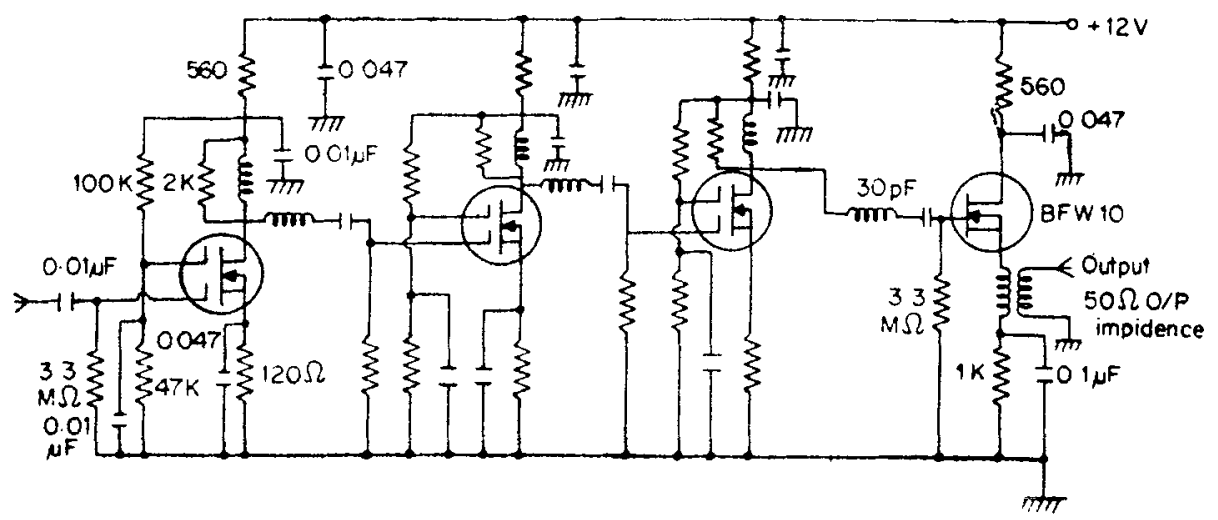

Figure 6. MOSFET (40673) tuned amplifier (gain $=60 \mathrm{~dB}, B W=2 \mathrm{MHz}$ ). 
minated by using series tuned resonant circuits between the stages. The total gain of the tuned amplifier is $\sim 60 \mathrm{~dB}$ and the recovery time is about $12 \mu \mathrm{sec}$. The MOSFET's used have a high input impedance $\sim 10^{14} \mathrm{ohms}$, and low noise characteristics, $\sim 3 \mathrm{~dB}$. The $2 \mathrm{~K}$ resistor across the inductance in the drain circuit is used for damping the $Q$ of the coils. With high $Q$ coils the circuit tends to oscillate.

The recovery time has been reduced to $\sim 7 \mu$ sec using the quench circuit described later.

2.4c. Phase shifter and the phase sensitive detector. (Figure 7) Generally, the signal has a different phase with respect to the reference. The $\mathrm{S} / \mathrm{N}$ is a maximum when the signal is in phase with the reference. A phase shifter is included in the reference channel to provide the necessary phase change. The second output of the power divider (figure 2) is used as the input ( $1 \mathrm{~V}$ maximum) to a Brüker broadband phase shifter, BP-4 (1-100 MHz, $0-360^{\circ}$ phase adjust), after suitable attenuation. The phase shifter output is connected to the reference input of the phase detector.

A Hewlett-Packard, HP 10534A, double-balanced mixer is used as the phase sensitive detector. The output of the phase shifter is connected to the R-port and the tuned amplifier output (i.e. the signal) is connected to the L-port. The X-port gives the detected output signal.

2.4d. Video amplifier. The detected signal at the output of the PSD requires further amplification before it could be displayed on an oscilloscope. As mentioned earlier, the preamplifier circuit is used as the first stage of the video amplifier (figure 8). The signal is fed to this circuit through an RC filter. A Motorola MC 1445 (a gated video amplifier) is used as the second stage of the video amplifier with a suitable time constant for filtering the noise. This IC also gives $\sim 20 \mathrm{~dB}$ gain and a bandwidth of $15 \mathrm{MHz}$. The total gain of the video amplifier is $\sim 40 \mathrm{~dB}$.

2.4e. Boxcar integrator. Although signals at the output of the video amplifier can be recorded and measured using a storage oscilloscope, the accuracy is limited in cases where the signal-to-noise ratio is small. In such cases, one looks for a signal averaging instrument. Boxcar integrators are commonly used in retrieval of transient signals buried in noise. It has two modes of operation-the scan mode and the single point mode, the former being used for wave form retrieval and the latter for amplitude measurement at a point of interest. Single point measurements are quite adequate for the measurement of spin relaxation times and a boxcar integrator

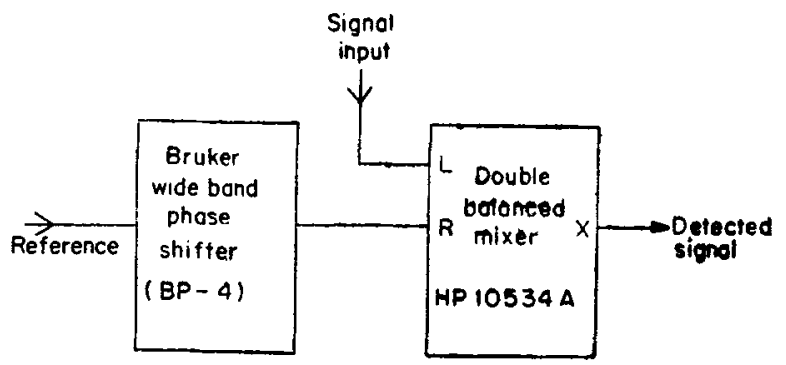

Figure 7. Phase shifter and phase sensitive detector. 


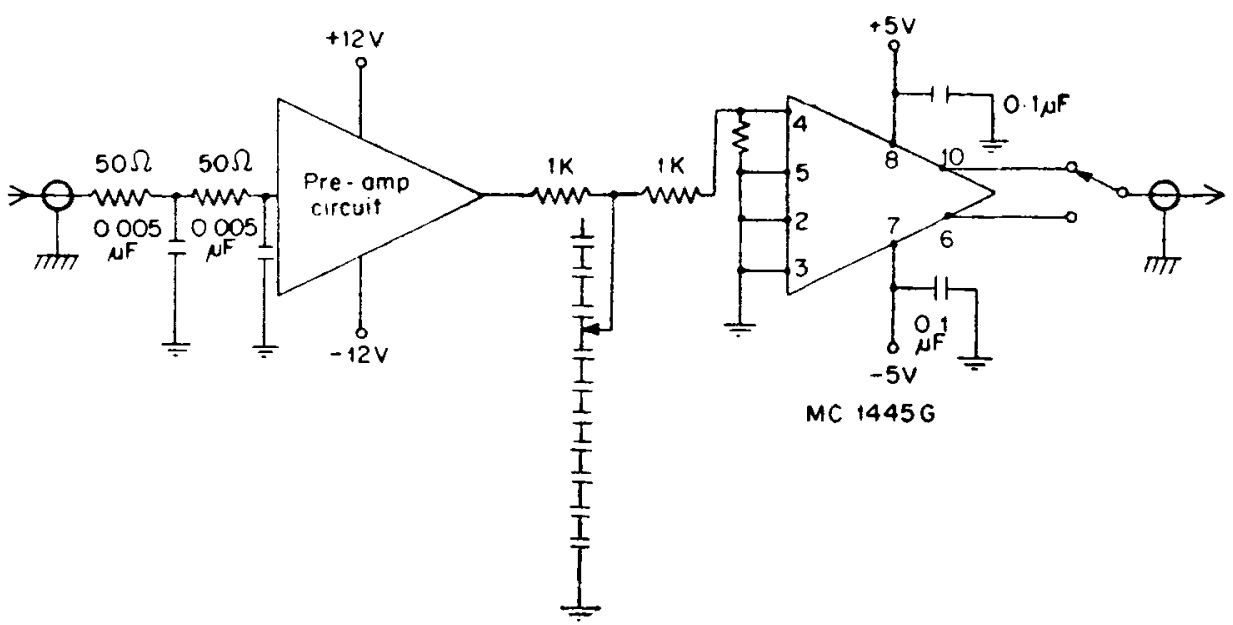

Figure 8. Video amplifier.

operating in this mode has been designed and constructed. The circuit details have already been published (Shenoy et al 1979).

Essentially, it has two channels of gated integrators, a differential amplifier, an A/D converter and a totaliser with digital display. The availability of a second channel extends its use as a differential boxcar, for measuring the difference in amplitudes of two signals. It can also be used as a double boxcar for measuring the signal amplitude in one channel and the baseline noise in the other channel. The double boxcar mode has the advantage of overcoming the problem of baseline instabilities.

The sensitivity of the boxcar integrator is better than $2 \mathrm{mV}$. The linearity is about $0.1 \%$ and signals of both polarities can be measured.

\subsection{Pulsed NMR probe}

The probe is yet another vital part of the spectrometer. A single coil series tuned resonant circuit of Clark and McNeil (1973) has been used as the pulsed NMR probe (in figure 9) which permits both efficient coupling of the transmitter power to the sample coil and good signal reception with a high signal/noise ratio into the receiver. The probe consists of three tuned sub-systems - the transmitter output circuit $\left(R_{1}\right.$, $\left.L_{1}, C_{1}\right)$, the sample coil circuit $\left(R_{2}, L_{2}, C_{2}\right)$ and the receiver input circuit $\left(R_{3}, R_{i n}\right.$, $\left.C_{i n}, C_{3}, L_{3}\right)$. When each circuit is tuned to the same frequency $W\left(=2 \pi \times 10^{7}\right.$ radians/ sec) it represents a high $Q$ circuit. The diode pairs $(2 \times 1 \mathrm{~N} 914) D_{1}-D_{2}$ and $D_{3}-$ $D_{4}$, to a good approximation, appear as short circuits to the transmitter pulse and as open circuits to the subsequent small FID signal induced in $L_{2}$.

For the transmitter part of the probe, the design equations (Clark and MeNeil 1973, equations (7), (9) and (10)) have been used with the following parameters: $\omega=6 \times 10^{7} \mathrm{rad} \mathrm{sec}^{-1}(10 \mathrm{MHz})$, output impedance $R_{0}=75 \mathrm{ohms}$, ring down time $\tau=3 \times 10^{-7} \mathrm{sec}$ and $Q=9$. The calculated values of $L_{1}$ and $L_{2}$ are $0.28 \mu H$ and 0.56 $\mu H$. The tuning condition yields $C_{1} \sim 1000 \mathrm{pf}$ and $C_{2} \sim 500 \mathrm{pf}$. The value of $Q_{T}$ (Clark and McNeil 1973, equation (1)) requires $R_{1}+R_{2} \sim 5$ ohms. A discrete resistor has been added at $R_{1}$ to achieve the above value of $Q_{T}$. 


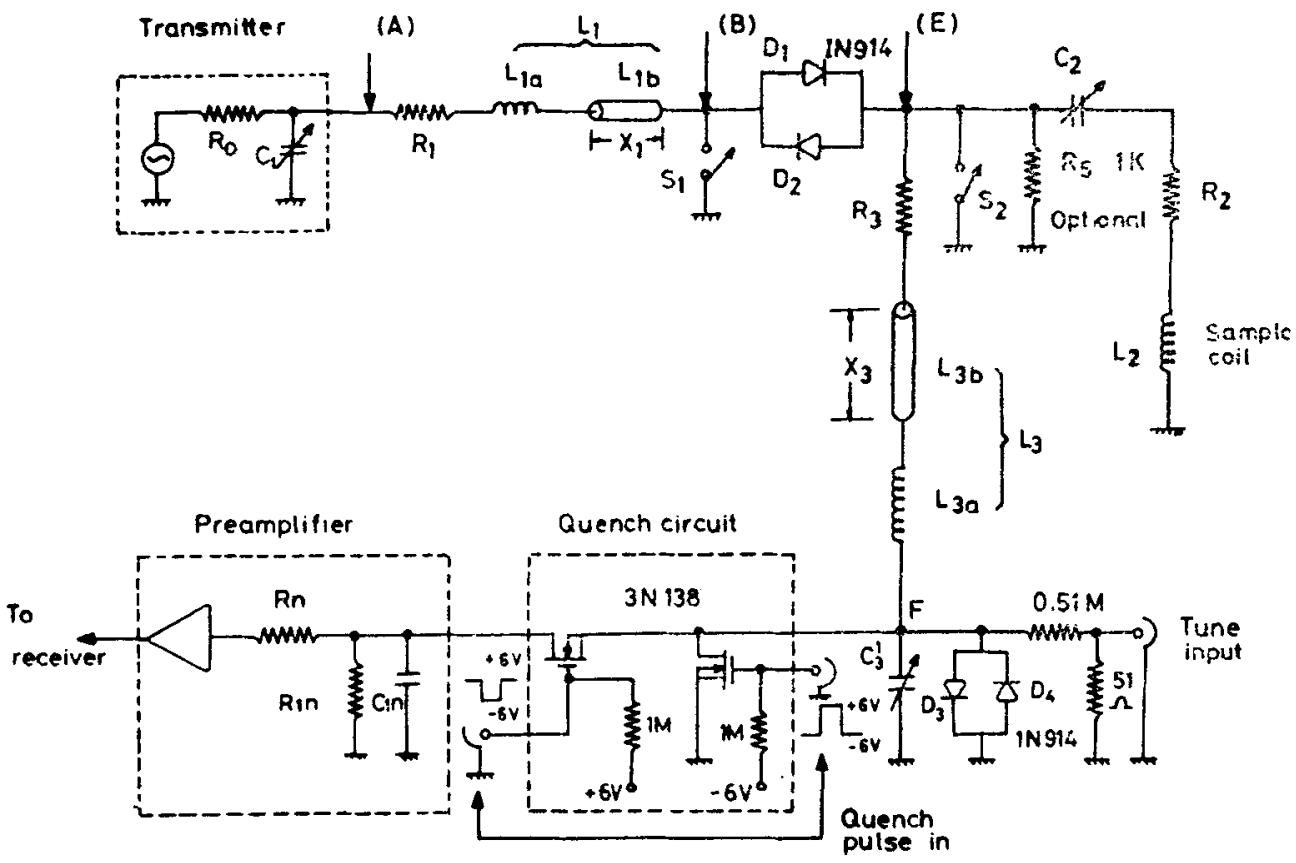

Figure 9. Pulsed NMR probe.

For the signal reception circuit of the probe, the design equations (Clark and McNeil 1973, equations (24) and (30)) require $Q_{2}\left(=\omega L_{2} / R_{2}\right)$ and $Q_{3}\left(=\omega L_{3} / R_{3}\right)$ to be large. Reasonable values of $Q_{2}(\sim 50)$ and $Q_{3}(\sim 100)$ (the restricted geometry of the sample coil often dictates a smaller $Q$ for it than for $L_{3}$ ) have been chosen. The optimum value of $\mathrm{S} / \mathrm{N}$ is obtained when $L_{3} \sim L_{2} / 2$ which yields about $84 \%$ of the $\mathrm{S} / \mathrm{N}$ ratio of the ideal value.

For an appreciable $\mathrm{S} / \mathrm{N}, Q_{2}$ and $Q_{3}$ of $L_{2}$ and $L_{3}$ respectively, should be made as large as possible. But considering the homogeneities of $H_{0}$ and $H_{1}$, the dimension of $L_{2}$ has been restricted. A coil of 9 turns of 18 SWG copper wire is closely wound on a $10 \mathrm{~mm}$ glass sample tube for optimum homogeneity in $H_{1}$.

2.5a. Recovery time improvement. The probe circuit has a recovery time of $\sim 12 \mu \mathrm{sec}$. To reduce the recovery time, a series and shunt quench circuit (figure 10) has been included before the preamplifier. Two RCA MOSFETS (3N138), which have a low ON resistance $(\sim 200$ ohms $)$ and a high OFF resistance $\left(\sim 10^{13}\right.$ ohms $)$, have been used as choppers. Normally, the series chopper is kept $O N$ and the shunt chopper OFF by having $+6 \mathrm{~V}$ and $-6 \mathrm{~V}$ on their gates respectively. During the transmitter pulse ON time, the synchronous pulses (amplified to 6 to $-6 \mathrm{~V}$, figure 10) from the pulse programmer switch the shunt chopper $O N$ and the series chopper OFF. The transmitter pulses are grounded by the shunt chopper and any leakage is blocked by the series chopper. The reduction of leakage of the transmitter pulses to the preamplifier reduces the recovery time to $\sim 7 \mu$ sec. The recovery time may be further improved by controlling the fall time of the quench pulses. The series and shunt choppers turn $O N$ and OFF respectively, during the OFF time of the synchronous pulses. During this time the FID signal is processed by the receiver. 


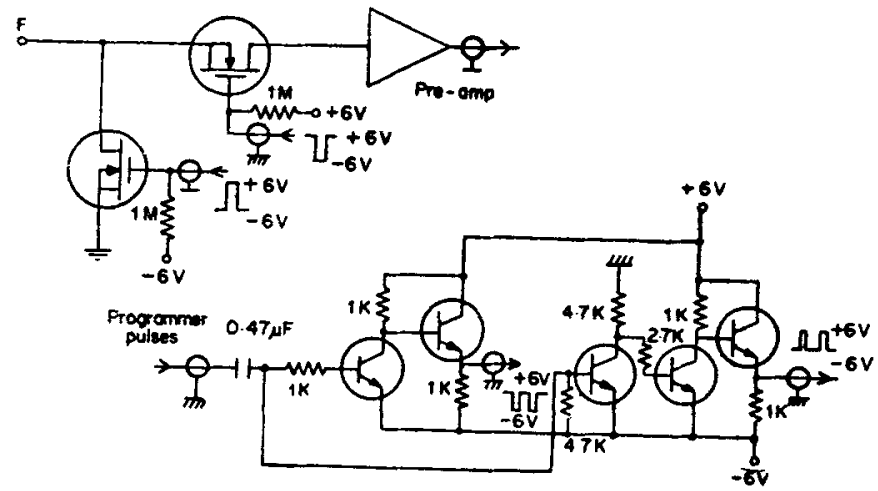

Figure 10. Quench circuit (3N138 MOSFETS) and the pulse amplifier (All transistors are $2 \mathrm{~N} 2369$ )

\subsection{Power supplies}

Separate regulated power supplies have been used for each unit of the spectrometcr. All low tension power supplies have been constructed using RCA CA3085A voltage regulator, while the power supply for the power stage of the transmitter has been constructed using vacuum tube circuits.

\section{Conclusions}

The above spectrometer has been in operation for the last three years and has been working satisfactorily. The spectrometer described is designed for spin lattice relaxation measurements in the laboratory frame. Incorporation of a few additions such as gated RF in separate channels for individual pulses with necessary phase shifters, and separate control for the amplitude of RF pulses, etc., extends the use of the spectrometer for $T_{1} \rho, T_{1 D}$ measurements. Inclusion of a multichannel signal averager in place of the boxcar integrator would make the spectrometer suitable for sophisticated experiments involving more than one relaxation component.

\section{References}

Adduci D J, Hornung P A and Torgeson D R 1976 Rev. Sci. Instrum. 471174

Clark W G and McNeil J A 1973 Rev. Sci. Instrum. 44844

Farrar T C and Becker E D 1971 Pulse and Fourier transform NMR (New York: Academic Press)

Hahn E L 1950a Phys. Rev. 77297

Hahn E L 1950b Phys. Rev. 80580

Lowe I J and Tarr C E 1968 J. Phys. E1 604

Ramadan B, Ng T C and Tward E 1974 Rev. Sci. Instrum., 451174

Shenoy R K and Ramakrishna J 1975 Symposium on Instrumentation and Measurements, Cálcutta Shenoy R K, Ramakrishna J and Srinivasan R 1976 J. Phys. E9 779

Shenoy R K and Ramakrishna J 1977 Nucl. Phys. Solid State Phys. (India) 20C 655

Shenoy R K and Ramakrishna J 1979 Symposium of All India Magnetic Resonance Spectroscopists Hyderabad

Shenoy R K, Ramakrishna J and Jeffrey K R 1979 Pramana 131 